\title{
In situ viscometry by optical trapping interferometry
}

\author{
Guzmán, C.; Flyvbjerg, Henrik; Köszali, R.; Escoffet, C.; Forró, L; Jeney, S.
}

\section{Published in:}

Applied Physics Letters

Link to article, DOI:

$10.1063 / 1.3020713$

Publication date:

2008

Document Version

Publisher's PDF, also known as Version of record

Link back to DTU Orbit

Citation (APA):

Guzmán, C., Flyvbjerg, H., Köszali, R., Escoffet, C., Forró, L., \& Jeney, S. (2008). In situ viscometry by optical trapping interferometry. Applied Physics Letters, 93(18), 184102. https://doi.org/10.1063/1.3020713

\section{General rights}

Copyright and moral rights for the publications made accessible in the public portal are retained by the authors and/or other copyright owners and it is a condition of accessing publications that users recognise and abide by the legal requirements associated with these rights.

- Users may download and print one copy of any publication from the public portal for the purpose of private study or research.

- You may not further distribute the material or use it for any profit-making activity or commercial gain

- You may freely distribute the URL identifying the publication in the public portal

If you believe that this document breaches copyright please contact us providing details, and we will remove access to the work immediately and investigate your claim. 


\title{
In situ viscometry by optical trapping interferometry
}

\author{
Camilo Guzmán, ${ }^{1}$ Henrik Flyvbjerg, ${ }^{2}$ Roland Köszali, ${ }^{1}$ Carole Ecoffet ${ }^{3}$ László Forró, ${ }^{1}$ and \\ Sylvia Jeney ${ }^{1, a)}$ \\ ${ }^{1}$ Institut de Physique de la Matière Complexe, Ecole Polytechnique Fédérale de Lausanne (EPFL), \\ CH-1015 Lausanne, Switzerland \\ ${ }^{2}$ Department of Micro-and Nanotechnology, Technical University of Denmark (DTU), \\ DK-2800 Kongens Lyngby, Denmark \\ ${ }^{3}$ Université Haute Alsace, ENSCMu, CNRS UMR 7525, Dept Photochim Gen, F-68093 Mulhouse, France
}

(Received 16 September 2008; accepted 13 October 2008; published online 4 November 2008)

\begin{abstract}
We demonstrate quantitative in situ viscosity measurements by tracking the thermal fluctuations of an optically trapped microsphere subjected to a small oscillatory flow. The measured power spectral density of the sphere's positions displays a characteristic peak at the driving frequency of the flow, which is simply proportional to the viscosity, when measured in units of the thermal power spectral density at the same frequency. Measurements are validated on different water-glycerol mixtures, as well as in a glycerol gradient, where no a priori knowledge of the solution is used to determine the glycerol concentration. (C) 2008 American Institute of Physics. [DOI: 10.1063/1.3020713]
\end{abstract}

The two main mechanical properties of a complex fluid are its elasticity and viscosity. Understanding and quantitative characterization of viscoelasticity in small volumes such as cells or microfluidic devices require the development of methods that can access these parameters in situ down to the nanoscale. Their measurement with optical tweezers was demonstrated qualitatively in a living yeast cell. ${ }^{1}$ They have, in general, great potential for single-cell diagnosis, as for instance, viscoelastic cell deformability is sensitive enough to monitor changes occurring during cell alteration from a normal toward a cancerous state. ${ }^{2}$ Changes in viscosity play a role in the regulation of diffusion-mediated processes, such as intercellular communication, signaling, and differentiation. Furthermore, it is a critical factor in the optimization of liquid photoresists used for stereolithography in microfabrication processes. ${ }^{3}$

To demonstrate quantitative in situ viscosity measurements, we here probe local viscosities with the thermal fluctuations of an optically trapped microsphere embedded in the fluid of interest. The sphere's position is recorded with a quadrant photodiode by back-focal plane interferometry. ${ }^{4,5}$ Several similar so-called active microrheological methods have been introduced before, ${ }^{6-11}$ but they all rely on calibration against standards, for a preliminary determination of the detector's sensitivity or of the local laser power at the sample, employing usually reference solutions with wellknown properties. Therefore, they suffer limitations when truly quantitative in situ measurements are needed. A recently introduced detector calibration procedure ${ }^{12,13}$ is independent of such a priori assumptions on fluid properties and detector sensitivity. Instead, it measures them by considering the power spectrum of a trapped Brownian sphere exposed to a Stokes' drag arising from a small oscillatory fluid flow. To validate the proposed method for in situ viscosity measurements, aqueous glycerol solutions at different concentrations are used as typical purely viscous model fluids of dynamic viscosity $\eta$ and density $\rho$. A suspension of $10^{6}$ microspheres/ml fluid is loaded into a liquid chamber (size

\footnotetext{
a) Author to whom correspondence should be addressed. Electronic mail: sylvia.jeney@epfl.ch.
}

$\approx 20 \times 5 \mathrm{~mm}^{2}$ and thickness $\sim 100 \mu \mathrm{m}$ ) by capillarity. Subsequently, the liquid cell is sealed with vacuum grease. A piezoelectric stage moves the sample chamber with a sinusoidal oscillation $x_{\text {drive }}(t)=A \sin \left(2 \pi f_{\text {drive }} t\right)$ at a given amplitude $A$ and frequency $f_{\text {drive }}$, while the laser trap remains fixed. This creates a fluid flow around the trapped microsphere. Experiments are performed at room temperature $\left(\approx 24{ }^{\circ} \mathrm{C}\right)$ with a polystyrene bead (radius $a=0.5 \mu \mathrm{m}$ ) trapped with a Nd:YAG laser beam $(\lambda=1064 \mathrm{~mm}, 500$ $\mathrm{mW})$. The Brownian sphere is typically positioned at a distance $h \simeq 50 \mu \mathrm{m}$ away from the glass surface to avoid any boundary effects. ${ }^{14,15}$ The equation of motion gives the positions of the bead in the lab-trap system as the sum of its thermal fluctuations and its response to imposed oscillations in fluid velocity, $x(t)=x_{\text {therm }}(t)+x_{\text {resp }}(t)$. Accordingly, the resulting power spectral density $P(f)=\left\langle|\widetilde{x}(f)|^{2}\right\rangle / t_{\mathrm{msr}}$, with $t_{\mathrm{msr}}$ the measurement time, splits up into two components; $P(f)$ $=P_{\text {therm }}(f)+P_{\text {resp }}(f) .{ }^{12}$ To increase statistics, data acquisition is done at a high sampling rate of $200 \mathrm{kHz}$ during $t_{\mathrm{msr}}$ $=50 \mathrm{~s}$. At this $5 \mu$ s time resolution, Einstein's theory of Brownian motion is insufficient since it neglects the particle's inertia and hydrodynamic memory effects. As shown previously, ${ }^{16}$ ignoring such memory effects leads to errors in the determination of the trap stiffness $k$ and detector sensitivity $\beta$ at high sampling rates, for large particles, and strong traps. Under the present experimental conditions $(k$ $>30 \mu \mathrm{N} / \mathrm{m}$ ), neglecting inertia would lead to an underestimation of $k$ and $\beta$ of more than $15 \%$. We therefore use the hydrodynamically correct theory of Brownian motion, ${ }^{12,15}$ and hence have for $t_{\mathrm{msr}} \rightarrow \infty$ that

$$
P_{\text {resp }}(f)=\frac{\frac{1}{4} A^{2} f_{\text {drive }}^{2}\left[1+\left(f / f_{\nu}\right)^{1 / 2}\right]}{\left(f_{c}-\frac{f^{3 / 2}}{f_{\nu}^{1 / 2}}-\frac{f^{2}}{f_{m}}\right)^{2}+\left(f+\frac{f^{3 / 2}}{f_{\nu}^{1 / 2}}\right)^{2}} \delta\left(f-f_{\text {drive }}\right),
$$

i.e., a delta function spike at the driving frequency of the stage, and 


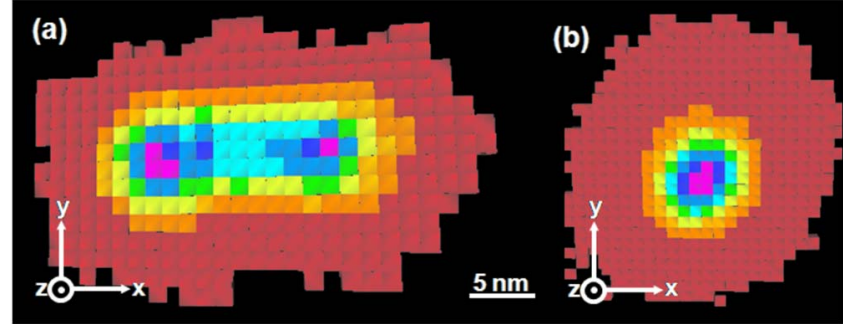

FIG. 1. (Color online) (a) The superposition of Brownian motion and the applied oscillations become visible in the particle's two-dimensional position histogram, when immersed in a solution of high viscosity such as $80 \%$ glycerol in $\mathrm{H}_{2} \mathrm{O}(\mathrm{w} / \mathrm{w})$. (b) If the viscosity is low, such as in pure $\mathrm{H}_{2} \mathrm{O}$, and the trap is strong enough, the bead's fluctuations will remain centered around the trapping potential's minimum.

$$
P_{\text {therm }}(f)=\frac{k_{B} T}{12 \pi^{3} \eta a} \frac{\left[1+\left(f / f_{\nu}\right)^{1 / 2}\right]}{\left(f_{c}-\frac{f^{3 / 2}}{f_{\nu}^{1 / 2}}-\frac{f^{2}}{f_{m}}\right)^{2}+\left(f+\frac{f^{3 / 2}}{f_{\nu}^{1 / 2}}\right)^{2}},
$$

describing the "thermal background" arising from Brownian fluctuations. The characteristic frequencies $f_{m}=3 \eta a / m, f_{\nu}$ $=\eta /\left(2 \pi a^{2} \rho\right)$, and $f_{c}=k /\left(12 \pi^{2} \eta a\right)$, with $m$ as the particle's mass and $k$ as the trap stiffness, are related to the particle, the fluid, and the trap, respectively. In practice, to avoid leakage, $t_{\mathrm{msr}}$ is chosen to be an integer multiple of $f_{\text {drive }}$ so that the discrete experimental power spectrum $P(f)$ features a Kronecker delta peak at $f=f_{\text {drive }}$ (Ref. 12, Appendix C). The viscosity of the fluid follows then directly from Eqs. (1) and (2),

$$
\eta=\frac{k_{B} T\left[P\left(f_{\text {drive }}\right)-P_{\text {therm }}\left(f_{\text {drive }}\right)\right]}{3 \pi^{3} a A^{2} f_{\text {drive }}^{2} P_{\text {therm }}\left(f_{\text {drive }}\right) t_{\text {msr }}} .
$$

The oscillating fluid imposes a viscous drag $\gamma=6 \pi \eta a$ on the trapped particle, resulting in an oscillating displacement of its Brownian fluctuations about the trap's center. Due to the laser counteracting on the particle's oscillations, the amplitude $A_{\text {resp }}$ of its response will be smaller than $A$ and depends on $\gamma$. To keep $A_{\text {resp }}$ within the linear range of the detector, ${ }^{5}$ we set $A=200 \mathrm{~nm}$, and $f_{\text {drive }}=10 \mathrm{~Hz}$ is chosen well below the resonance frequency of the piezostage. Furthermore, relatively strong trapping forces, i.e., highest possible $f_{c}$ (typically between 1 and $1000 \mathrm{~Hz}$ ), are adjusted by the incoming laser power to counterbalance the viscous drag on the bead. For a solution with high viscosity, for example, $80 \%(\mathrm{w} / \mathrm{w})$ glycerol in water, the particle's displacement with respect to the trapping potential minimum can be clearly detected in the two-dimensional histogram of the probability density of the particle's position [Fig. 1(a)]. In a solution with relatively low viscosity, such as pure water, the sphere's sinusoidal oscillations are masked by thermal fluctuations [Fig. 1(b)].

The measured $P(f)$ is more sensitive to $\gamma$ and features a characteristic spike at $f=f_{\text {drive, }}$, as can be seen in Fig. 2(a). At $f \neq f_{\text {drive }}$, Eq. (2) fits the blocked values with an accuracy better than $6 \%$ between $100 \mathrm{~Hz}$ and $10 \mathrm{kHz}$, and even $2 \%$ between 10 and $100 \mathrm{kHz}$, yielding the corner frequency $f_{c}$ [Fig. 2(b)]. $P_{\text {therm }}\left(f_{\text {drive }}\right)$ is directly read off the fit in Fig. 2(b), and $P\left(f_{\text {drive }}\right)$ from the graph in Fig. 2(a), resulting in $\eta$ by Eq. (3). The peak amplitude scales with the glycerol concentration and hence $\eta$ (inset). As viscosity increases $f_{c}$ drops below $10 \mathrm{~Hz}$ [lightest gray plot for pure glycerol in
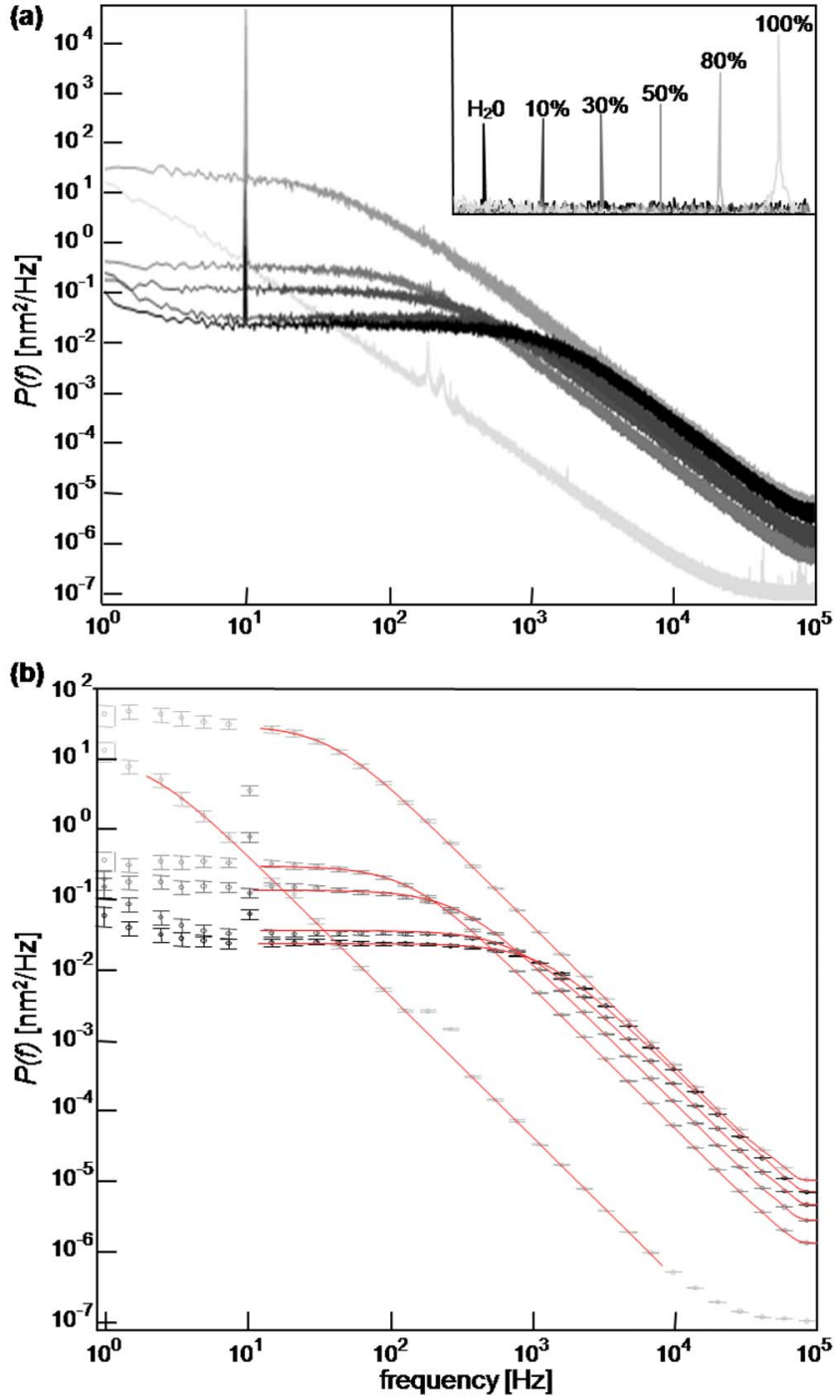

FIG. 2. (Color online) (a) Power spectral density $P(f)$ in different glycerol/ water fractions $(\mathrm{w} / \mathrm{w})$ varying from $100 \%$ to $0 \%$ glycerol (from light gray to black plots) with $A=200 \mathrm{~nm}$ and $f_{\text {drive }}=10 \mathrm{~Hz}$. Inset: zoom on the $10 \mathrm{~Hz}$ peaks to illustrate the differences in peak powers. For clarity, the peaks are shifted along both axes. Each curve is an average over $\sim 10$ consecutive measurements. (b) The raw $P(f)$ is blocked in 50 bins/decade and fitted by Eq. (2), taking aliasing into account (red continuous line). The error bars indicate the standard error on the mean.

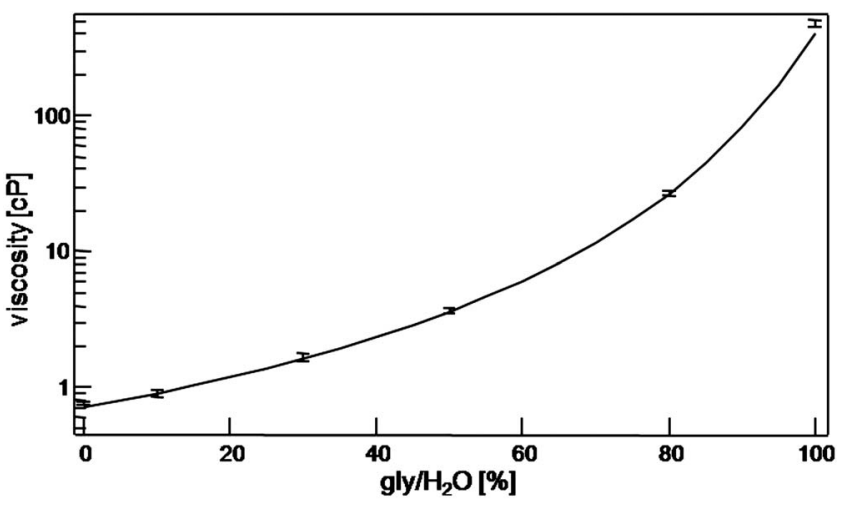

FIG. 3. Measured dynamic viscosity as a function of the glycerol concentration, fitted using Cheng's model (Ref. 17) (continuous line) with a temperature of $35^{\circ} \mathrm{C}$. The error bars show the standard error on the mean of $\sim 10$ consecutive measurements, based on the standard deviation of the same 10 measurements. 


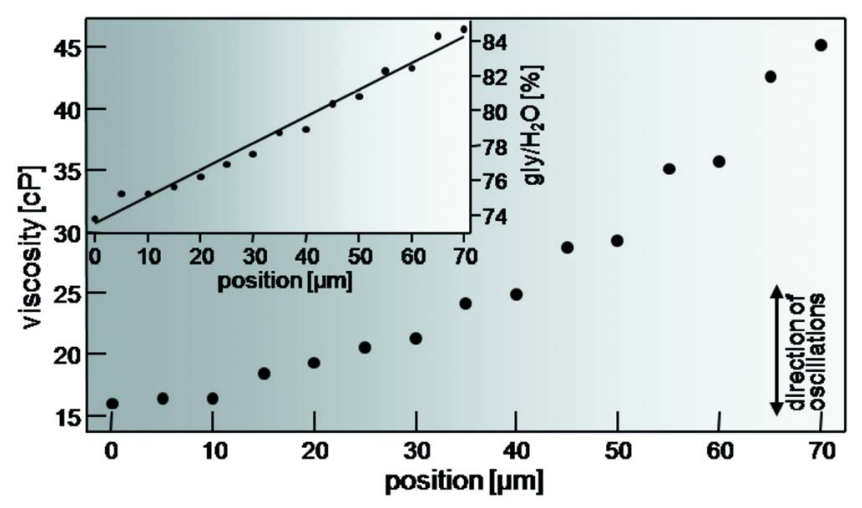

FIG. 4. (Color online) Viscosity measured at different positions along a concentration gradient of glycerol in water. Inset: concentration of glycerol, determined according to Ref. 17, as function of relative positions showing the linearity of the gradient.

Fig. 2(a)] even with highest trapping forces. The resulting $\eta$ is plotted in Fig. 3(a) as a function of the glycerol concentration. The continuous line represents an empirical formula recently introduced by Cheng. ${ }^{17}$ It fits our data best for a temperature of $\approx 35{ }^{\circ} \mathrm{C}$, pointing to laser-induced heating in the trapping focus. ${ }^{18}$ Finally, we tested the method in a nonhomogeneous but still purely viscous medium, namely, a concentration gradient of glycerol in water. We measured the viscosity locally at different positions along the gradient, moving in steps of $5 \mu \mathrm{m}$ (Fig. 4). The time interval between two consecutive measurements, from one position to the next, was less than $60 \mathrm{~s}$, ensuring a stable gradient on this timescale. As shown in the inset, the glycerol concentration at any position in our sample chamber can be determined, and also the linearity of the created gradient is verified.

In conclusion, the presented methodology is fast and can easily be adapted to study in quasireal-time minute volumes of even dynamic and inhomogeneous media, such as the cytoplasm. As viscosity of a fluid is directly related to its temperature, this method can also be used to quantify laser- induced heating in an optical trap in a medium of known viscosity, or more generally detect local temperature changes at the nanoscale.

S.J. and C.G. acknowledge the financial support from the Gebert Rüf Foundation (Contract No. P-012/06), the Swiss National Science Foundation for Nanoscale Science (NCCR), and EPFL for funding the experimental equipment. We thank F. Mor and P. Annibale for helpful discussions.

${ }^{1}$ I. M. Tolić-Nørrelykke, E.-L. Munteanu, G. Thon, L. Oddershede, and K. Berg-Sørensen, Phys. Rev. Lett. 93, 078102 (2004).

${ }^{2}$ J. Guck, S. Schinkinger, B. Lincoln, F. Wottawah, S. Ebert, M. Romeyke, D. Lenz, H. M. Erikson, R. Ananthakrishnan, D. Mitchell, J. Ks, S. Ulvick, and C. Bilby, Biophys. J. 88, 3689 (2005).

${ }^{3}$ R. S. Kane, Angew. Chem., Int. Ed. 47, 1368 (2008).

${ }^{4}$ M. Allersma, F. Gittes, M. J. deCastro, R. J. Stewart, and C. F. Schmidt, Biophys. J. 74, 1074 (1998).

${ }^{5}$ A. Pralle, M. Prummer, E.-L. Florin, E. H. K. Stelzer, and J. K. H. Hörber, Microsc. Res. Tech. 44, 378 (1999).

${ }^{6}$ M. T. Valentine, L. E. Dewalt, and H. D. Ou-Yang, J. Phys.: Condens. Matter 8, 9477 (1996).

${ }^{7}$ R. Ługowski, B. Kolodziejczyk, and Y. Kawata, Opt. Commun. 202, 1 (2002).

${ }^{8}$ B. A. Nemet, Y. Shabtai, and M. Cronin-Golomb, Opt. Lett. 27, 264 (2002).

${ }^{9}$ A. I. Bishop, T. A. Nieminen, N. R. Heckenberg, and H. RubinszteinDunlop, Phys. Rev. Lett. 92, 198104 (2004).

${ }^{10}$ G. Pesce, A. Sasso, and S. Fusco, Rev. Sci. Instrum. 76, 115105 (2005).

${ }^{11}$ S. J. Parkin, G. Knöner, T. A. Nieminen, N. R. Heckenberg, and H. Rubinsztein-Dunlop, Phys. Rev. E 76, 041507 (2007).

${ }^{12}$ S. F. Tolić-Nørrelykke, E. Schäffer, J. Howard, F. S. Pavone, F. Jülicher, and H. Flyvbjerg, Rev. Sci. Instrum. 77, 103101 (2006).

${ }^{13}$ E. Schäffer, S. F. Nørrelykke, and J. Howard, Langmuir 23, 3654 (2007).

${ }^{14}$ S. Jeney, B. Lukić, J. A. Kraus, T. Franosch, and L. Forró, Phys. Rev. Lett. 100, 240604 (2008).

${ }^{15}$ K. Berg-Sørensen and H. Flyvbjerg, Rev. Sci. Instrum. 75, 594 (2004).

${ }^{16}$ B. Lukić, S. Jeney, Z. Sviben, A. J. Kulik, E.-L. Florin, and L. Forró, Phys. Rev. E 76, 011112 (2007).

${ }^{17}$ N.-S. Cheng, Ind. Eng. Chem. Res. 47, 3285 (2008).

${ }^{18}$ E. J. G. Peterman, F. Gittes, and C. F. Schmidt, Biophys. J. 84, 1308 (2003). 